\title{
Molecular markers associated with perineural invasion in pancreatic ductal adenocarcinoma
}

\author{
JUNFENG ZHANG ${ }^{*}$, XUELIANG FU* , DEJUN LIU* , MINWEI YANG, JIANYU YANG, \\ YANMIAO HUO, WEI LIU, RONG HUA, YONGWEI SUN and JIAN WANG \\ Department of Biliary-Pancreatic Surgery, Ren Ji Hospital, School of Medicine, \\ Shanghai Jiao Tong University, Shanghai 200127, P.R. China
}

Received October 8, 2019; Accepted April 27, 2020

DOI: $10.3892 / \mathrm{ol} .2020 .11866$

\begin{abstract}
Perineural invasion (PNI) is a prominent characteristic of pancreatic ductal adenocarcinoma (PDAC). PNI is associated with tumor progression, local recurrence and neuropathic pain; therefore, the identification of biomarkers associated with PNI may be beneficial in assessing the prognosis for patients with PDAC. Using an in vivo model of PNI, five pancreatic cancer cell lines (PANC-1, CFPAC-1, CAPAN-2, SW1990 and ASPC-1) were divided into two groups: High-(comprising PANC-1, CFPAC-1 and CAPAN-2) and low PNI (comprising SW1990 and ASPC-1). Differentially expressed genes (DEGs) between the two groups were identified using the GSE26088 dataset, and were regarded as PNI-associated genes. A total of 445 DEGs associated with PNI (fold change $>1.5$ or $<0.66$; $\mathrm{P}<0.05$ ) were identified, which included 176 up- and 269 downregulated genes. Kyoto Encyclopedia of Genes and Genomes pathway enrichment analysis and function annotation were performed, and the NetworkAnalyst database was used for protein-protein interaction network analysis to identify hub genes. A total of 20 hub genes (gene degree, $\geq 6$ ) were identified. PNI was associated with the function 'chemokine signaling pathway'. The DEGs and hub genes were validated using the GSE102238 dataset and clinical tissue microarrays. Fibroblast growth factor 2 (FGF2) and catenin $\alpha 2$ were demonstrated to be associated with PNI using the GSE102238 dataset. Furthermore, clinical tissue microarray analysis demonstrated that FGF2 was associated with PNI and poor prognosis. The present study provided a potential method for the reliable identification of PNI-associated genes, although further investigation is required to validate these results.
\end{abstract}

Correspondence to: Dr Jian Wang, Department of Biliary-Pancreatic Surgery, Ren Ji Hospital, School of Medicine, Shanghai Jiao Tong University, 160 Pujian Road, Shanghai 200127, P.R. China

E-mail: dr_wangjianrenji@163.com

*Contributed equally

Key words: perineural invasion, pancreatic ductal adenocarcinoma, Kyoto Encyclopedia of Genes and Genomes pathway, hub gene, fibroblast growth factor 2

\section{Introduction}

Perineural invasion (PNI), which is characterized by the presence of cancer cells along nerves and/or within the epineurial, perineurial and endoneurial spaces of the neuronal sheath (1), have been reported in numerous types of cancer, including pancreas, colon, rectum and prostate $(1,2)$. Among these types of cancer, pancreatic ductal adenocarcinoma (PDAC) is associated with the highest rate of incidence of PNI. According to our previous meta-analysis (3) from 1998 to 2012, the overall incidence of PNI was $78.8 \%$ (range: $50.9-91.9 \%$ ). PNI is also associated with recurrence and poor prognosis (3). In several cases PNI may be accompanied by pain, and a number of molecules involved PNI are implicated in pain generation, such as nerve growth factor (4). Targeting these molecules may alleviate pain and decrease PNI, which could potentially improve treatment outcomes (2). A previous study (5) demonstrated that the nervous system participates in all stages of PDAC, including those that precede the appearance of cancer; which indicates that early interventions targeting the peripheral nervous system may present a novel strategy for treatment and the early diagnosis of PDAC.

To identify molecular markers associated with PNI, global gene expression level profiling has been used to investigate differentially expressed genes (DEGs) in nerve-invading and non-invading types of cancer cells. Koide et al (6) compared the gene expression level profiles between five pancreatic cancer cell lines (CAPAN-1, CAPAN-2, HPAFII, SW1990 and ASPC-1) with a high or low frequency of PNI. It was reported that $\mathrm{CD} 74$ is a candidate molecule involved in perineural invasion. Using an ex vivo model of PNI, Abiatari et al (7) distinguished highly nerve-invasive and non-invasive cancer cell clones in three pancreatic cancer cell lines (Panc-1, Colo357 and T3M4). Genome-wide transcriptome analyses identified several DEGs between the two cancer cell clones, which included kinesin family member 14 (KIF14) and Rho-GDP dissociation inhibitor $\beta$ (ARHGDI $\beta$ ). In the present study, gene expression profiling was used to identify changes in gene expression levels associated with PNI in five pancreatic cancer cell lines. In addition, Kyoto Encyclopedia of Genes and Genomes (KEGG) pathway enrichment analysis and functional annotation, and protein-protein interaction (PPI) network analysis were performed to identify hub genes 
associated with PNI. The results were verified using the GSE102238 dataset. The tissue microarray demonstrated that fibroblast growth factor 2 (FGF2) was associated with PNI in pancreatic cancer. It was hypothesized that the DEGs associated with PNI could identify molecular alterations and potential therapeutic targets for PDAC.

\section{Materials and methods}

Cell culture. The human ASPC-1, CAPAN-2, CFPAC-1, HPAC, PANC-1 and SW1990 pancreatic cancer cell lines were obtained from the Cell Resource Center of Shanghai Institute of Life Sciences, Chinese Academy of Sciences. The normal human pancreatic duct epithelial (HPDE) cell line was a gift from Professor Zhigang Zhang (State Key Laboratory of Oncogenes and Related Genes, Shanghai Cancer Institute, Shanghai Jiao Tong University, Shanghai, China). All cells were maintained in the indicated medium supplemented with 10\% (v/v) FBS (Stemcell Technologies, Inc.) and $1 \%$ antibiotics (penicillin and streptomycin) at $37^{\circ} \mathrm{C}$ in a humidified incubator with 5\% $\mathrm{CO}_{2}$. DMEM medium (Gibco; Thermo Fisher Scientific, Inc.) was used for HPDE, PANC-1 and SW1990; RPMI-1640 medium (Gibco; Thermo Fisher Scientific, Inc.) was used for ASPC-1, CAPAN-2 and HPAC; and Iscove's Modified Dulbecco's Medium (Gibco; Thermo Fisher Scientific, Inc.) was used for CFPAC-1.

In vivo model of PNI. All animal experiments were performed in accordance with the NIH Guide for the Care and Use of Laboratory Animals and approved by the Research Ethics Committee of East China Normal University (approval no. 20141204). The in vivo PNI model was established as previously described (8). Briefly, 4-week-old nude athymic mice [Balb/c; 17 male and 18 female, supplied by the jsj lab (www. jsj-lab.com)] were anesthetized using isoflurane (induced with $3 \%$ isoflurane and maintained using $1.5 \%$ isoflurane), and the right sciatic nerve was exposed. Pancreatic cancer cells were injected into the periphery of the sciatic nerve. Micro-injection of $5 \mu \mathrm{l}$ cell suspension at a concentration of $1 \times 10^{5} \mathrm{cell} / \mathrm{s} / \mathrm{ml}$ was performed using a $10 \mu \mathrm{l}$ microsyringe (Gaoge, Shanghai). A total of 35 mice were randomly divided into seven groups used ( $\mathrm{n}=5$ for each group). Mice were maintained in a specific pathogen-free animal laboratory and kept in a room at $18-23^{\circ} \mathrm{C}$ with $40-60 \%$ humidity, with free access to food and water on a $12 \mathrm{~h}$ day/night cycle. Intra-cage ammonia levels were maintained at 25 parts per million. The variables mainly included those that assessed the severity of PNI, including limb function and sciatic nerve function index. As the general condition and tumor size of the mice, especially the latter, may affect PNI, the mouse weight and tumor volume were also measured. All variables were measured weekly for 7 weeks. The tumor volume was calculated as $\mathrm{V}=(\mathrm{LxWxW}) / 2$, where $\mathrm{V}$ is the tumor volume, $\mathrm{L}$ is the tumor length and $\mathrm{W}$ is the tumor width. Limb function and sciatic nerve function index were measured to assess the severity of PNI (8). Limb function was graded according to the severity of the limb paralysis from 4 (normal) to 1 (total paw paralysis), according 3 (slight paralysis) and 2 (severe paralysis). The mice were lightly touched to stimulate movement and the limb function was measured. The sciatic nerve function index was calculated as the difference in spread length between the first and fifth toes of the hind limbs of the mouse before and after tumor invasion. Prior to tumor invasion, the limb can stretch and the spread length was considered to be normal. When the sciatic nerve was invaded by the tumor, the limb contracted and the spread length was decreased. Spread length was measured using vernier caliper.

After 7 weeks, animals were sacrificed using an overdose of $\mathrm{CO}_{2}$. Briefly, a flow rate of $4 \mathrm{l} / \mathrm{min}$ was used to displace $40 \%$ of the total cage volume, which resulted in insensibility before exposure to fatal concentrations $(<40 \%)$. The mice were exposed for $5 \mathrm{~min}$ and death was confirmed by observing the lack of respiration and cardiovascular movements in a room with normal air ventilation for at least $10 \mathrm{~min}$. Mouse tumors and nerve samples were collected in slices $(5 \mu \mathrm{m})$ to investigate the severity of nerve invasion. The frequency of PNI was analyzed according to the extent of sciatic nerve involvement, which was assessed via gross observation and immunohistochemistry.

Data collection. The microarray expression dataset (GSE26088) was obtained from the Gene Expression Omnibus (GEO) (ncbi.nlm.nih.gov/geo/), which includes 20 pancreatic cancer cell lines (9). The data of the five cell lines were adopted and divided into two groups according to their PNI ability. The expression data of raw CEL files were normalized, $\log 2$ transformed and background adjusted utilizing a Bioconductor package Robust MultiArray Average (RMA) through R 3.2.0 software (10). The normalized data were then analyzed using linear models for microarray data (version 3.26.8) and a modified t-test incorporating the Benjamini-Hochberg multiple hypotheses correction technique.

GSE102238 (11), which was also downloaded from the GEO database, was used as the verification dataset to confirm the DEG results. GSE102238 contains 100 samples, including 50 PDAC tumors and 50 matching adjacent non-cancerous tissue samples obtained from resected pancreas. Samples were divided into two groups according to PNI status stated in the dataset: PNI ( $\mathrm{n}=28$ pairs) and non-PNI ( $\mathrm{n}=22$ pairs). Normalization and analysis of data were also performed.

Functional enrichment analysis of DEGs. The criteria for selection of PNI-associated DEGs were: i) Fold-change $>1.50$ or $<0.66$; ii) $\mathrm{P}<0.05$. To determine the enriched biological Gene Ontology (GO) terms and pathways of the identified DEGs the Database for Annotation, Visualization and Integrated Discovery server (v6.8; david.ncifcrf.gov) was used, according to the standard enrichment computation method. $\mathrm{P}<0.05$ was selected as the cut-off value for enriched functions and pathways.

PPI network and subnetwork analysis. PNI-associated DEGs were uploaded into the NetworkAnalyst (http://www.networkanalyst.ca) database $(12,13)$ to determine the PPI network to identify hub genes. PPI network analysis included three steps. The first step is to identify genes or proteins of interest, such as differentially expressed genes. Secondly, these inputs (also known as seed proteins) were used to search and retrieve interactions from a curated PPI database. For each seed protein, a search algorithm was performed to identify proteins that directly interact with the seed proteins (first-order interactors). 


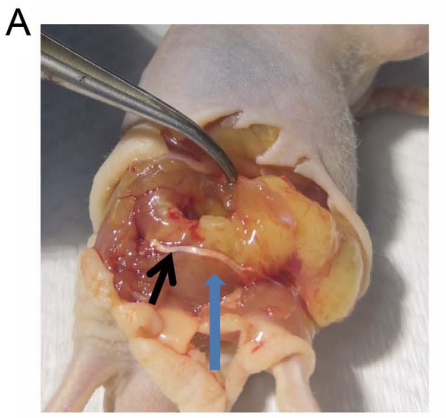

C

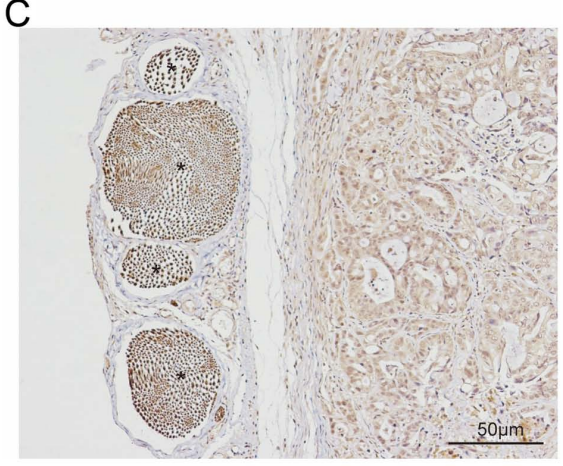

B

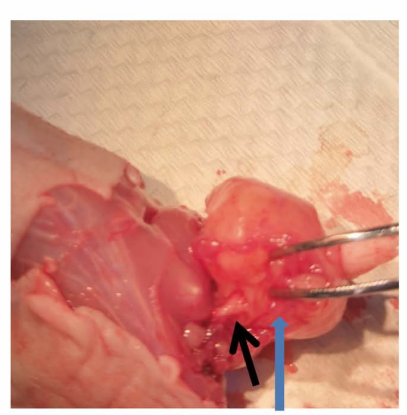

D

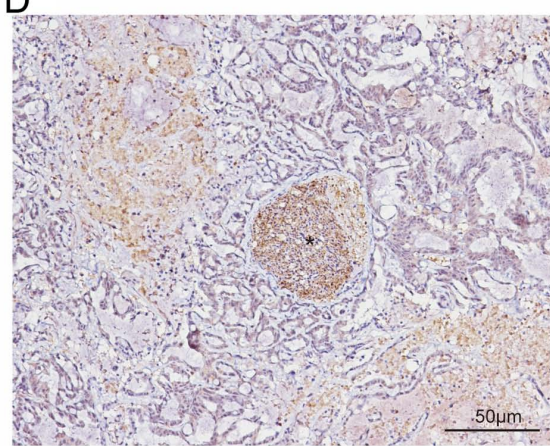

Figure 1. A total of two forms of nerve involvement in the PNI model in vivo. The sciatic nerve is located (A) superficial of the tumor tissue and (B) within the tumor tissue, providing evidence of PNI. Spatial association between sciatic nerve and tumor tissue, demonstrated by immunohistochemical staining of the pan-neuronal marker, PGP9.5. The sciatic nerve was located (C) superficial of or (D) within tumor tissue. The black arrow indicates the sciatic nerve; blue arrow indicates tumor tissue * indicates PGP9.5(+) nerve. PNI, perineural invasion.

If the default network constructed with first-order interactors is small, users can search for higher-order interactions. When there are too many seed proteins and the default network is too large to be visualized, users can choose to focus only on networks within these seed proteins (zero-order interactors). In the present study, only interacting pairs containing $\geq 1$ DEG were selected to construct the PPI network, with a confidence score $>0.90$, as the cut-off value. The third step is network analysis which included two approaches. The topology analysis search for important nodes (hub genes), which are useful as biomarkers or therapeutic targets. The results from network analysis was subsequently validated by other well-established approaches, including GO or KEGG pathway enrichment analysis $(12,13)$.

Clinical tissue microarrays. The Human PDAC tissue microarray containing 205 cases of tumor and matched non-tumor tissues, as described by Yang et al (14). Non-tumor tissue 1-2 cm away from the tumor tissue was used as the control, therefore it was difficult to ensure that non-tumor tissues were normal pancreatic tissues. It is possible that small portion of the non-tumor tissues were chronic pancreatitis tissues. Only patients with complete follow-up data for $>2$ years were included in the present study. The follow-up data included postoperative treatment, tumor marker and imaging examination, such as CT, MRI and PET-CT. In total, 12 cases, which were lost within two years after operation, were excluded. Tumors were classified using the Tumor-Node-Metastasis (TNM) classification criteria, according to the American Joint Committee on Cancer (8th edition) (15). Patients with TNM stage IV were excluded from the analysis $(n=13)$, as were patients who died of perioperative complications, such as serious pancreatic leakage, bleeding and systemic infections $(n=23)$. Finally, 157 cases with complete follow-up data were included. Tumor differentiation, nodal metastasis and lymphovascular invasion were determined according to pathological results. PNI was defined as PDAC cell infiltration of the perineural space between the perineurium and endoneurium of the peripheral nerve in directly contact with the endoneurium and intraneural invasion (6). The present study was approved by the Research Ethics Committee of Renji Hospital, School of Medicine, Shanghai Jiao Tong University (Shanghai, China). Written informed consent was provided by all patients prior to enrolment.

Immunohistochemistry (IHC) analysis. IHC staining was performed as previously described (16). Biomarker expression levels were detected using a commercially available rabbit polyclonal antibody against FGF2 (1:200; cat no. ab126861; Abcam). FGF2 expression levels were scored according to staining intensity and proportion. The intensity score was classified as 0 (no staining), 1 (weak staining), 2 (moderate staining) or 3 (strong staining). The proportion score, based on the percentage of tumor cells, was classified as $0(<5 \%), 1(6-35 \%), 2(36-70 \%)$ or $3(>70 \%)$. The final score was calculated using the proportion and the staining intensity scores and classified as follows: -, 0-1; ,$+ 2-3 ;++, 4-6$ and,$+++>6$. Low expression levels were defined as a total score $<4$; high expression levels were defined as a total score $\geq 4$. The low expression group included '-' and ' + ', while the high expression group included ' ++ ' and ' +++ '. The mouse monoclonal antibody against PGP9.5 (1:500; cat. no. ab8189; Abcam) was used as a pan-neuronal marker.

Reverse transcription-quantitative (RT-q)PCR. RT-qPCR was performed as previously described (14) using the aforementioned cell lines and GAPDH as an internal control. Data was 
Table I. Characteristics of two PNI ability group in vivo at 7 weeks.

\begin{tabular}{lcc}
\hline & \multicolumn{2}{c}{ PNI ability } \\
\cline { 2 - 3 } Variable & High $(\mathrm{n}=15)$ & Low (n=10) \\
\hline Cell line & PANC-1, CFPAC-1, CAPAN-2 & SW1990, ASPC-1 \\
Mean mouse weight, week 0, g \pm SD & $18.86 \pm 0.38$ & $19.00 \pm 0.39$ \\
Mean mouse weight, week 7, g \pm SD & $24.95 \pm 0.97$ & $25.58 \pm 0.74$ \\
Mean tumor length, mm \pm SD & $10.44 \pm 2.36$ & $10.66 \pm 2.40$ \\
Mean tumor width, mm \pm SD & $7.99 \pm 1.56$ & $8.62 \pm 2.06$ \\
Mean tumor volume, mm ${ }^{3} \pm$ SD & $365.35 \pm 193.48$ & 0.383 \\
Mean left SNFI, mm \pm SD & $9.67 \pm 0.36$ & 0.823 \\
Mean right SNFI, mm \pm SD & $7.10 \pm 1.76$ & 0.390 \\
Mean limb function \pm SD & $2.60 \pm 1.10$ & $0.46 \pm 0.25$ \\
\end{tabular}

${ }^{\mathrm{a}} \mathrm{P}<0.05$. PNI, perineural invasion; SNFI, sciatic nerve function index.

Table II. Top 10 up- and downregulated DEGs.

A, Upregulated DEGs

\begin{tabular}{llrr}
\hline Gene symbol & \multicolumn{1}{c}{ Description } & Fold-change & P-value \\
\hline BNIP3 & BCL2 interacting protein 3 & 5.58 & 0.001235 \\
EFEMP1 & EGF-containing fibulin-like extracellular matrix protein 1 & 3.73 & 0.006757 \\
GNB4 & G protein subunit $\beta$ 4 & 3.65 & 0.001084 \\
NETO2 & Neuropilin and tolloid-like 2 & 3.55 & 0.000477 \\
LY75 & Lymphocyte antigen 75 & 3.34 & 0.010767 \\
TSHZ3 & Teashirt zinc finger homeobox 3 & 3.24 & 0.001838 \\
LIMS3 & LIM zinc finger domain-containing 3 & 3.19 & 0.0000414 \\
TMSB15B & Thymosin $\beta$ 15B & 3.01 & 0.003074 \\
NAP1L5 & Nucleosome assembly protein 1-like 5 & 2.90 & 0.000186 \\
CLDN10 & Claudin 10 & 2.86 & 0.007955 \\
\hline
\end{tabular}

B, Downregulated DEGs

\begin{tabular}{llrr}
\hline SPON1 & Spondin 1 & 0.22 & 0.0000353 \\
CDH11 & Cadherin 11 & 0.22 & 0.0173247 \\
SDPR & Caveolae-associated protein 2 & 0.31 & 0.0000935 \\
SOSTDC1 & Sclerostin domain-containing 1 & 0.31 & 0.0012579 \\
LUM & Lumican & 0.33 & 0.0286506 \\
IL1B & Interleukin 1 $\beta$ & 0.35 & 0.0179449 \\
ESM1 & Endothelial cell specific molecule 1 & 0.36 & 0.0003328 \\
CCL20 & C-C motif chemokine ligand 20 & 0.36 & 0.0003672 \\
SULF2 & Sulfatase 2 & 0.36 & 0.0227548 \\
PDE4B & Phosphodiesterase 4B & 0.37 & 0.0005664
\end{tabular}

DEGs, differentially expressed genes.

quantified using the $2^{-\Delta \Delta \mathrm{Cq}}$ method (17). The primer sequences used were as follows: FGF2 forward, 5'-AGAAGAGCGACC CTCACATCA-3' and reverse, 5'-CGGTTAGCACACACT CCTTTG-3'; catenin $\alpha 2$ (CTNNA2) forward, 5'-GGACGC
TAACAGTGGAAAGG-3' and reverse, 5'-GAGTGGCTT GCTCTACAGAGG-3'; and GAPDH forward, 5'-GCATTG CCCTCAACGACCAC-3' and reverse, 5'-CCACCACCCTGT TGCTGTAG-3'. 
Table III. Top 5 Gene Ontology enrichment analysis of DEGs associated with PNI.

A, Upregulated DEGs

\begin{tabular}{llr}
\hline Sub-ontology & \multicolumn{1}{c}{ Terms } & P-value \\
\hline MF & Calcium ion binding & 0.017056 \\
& Transcription factor activity & 0.030121 \\
& $\beta$-tubulin binding & 0.036267 \\
& Protein heterodimerization activity & 0.041477 \\
BP & Cilium morphogenesis & 0.028336 \\
& Brown fat cell differentiation & 0.029846 \\
& Positive regulation of epithelial to mesenchymal transition & 0.031598 \\
& Cell death & 0.033012 \\
& Positive regulation of stress fiber assembly & 0.042919 \\
&
\end{tabular}

B, Downregulated DEGs

\begin{tabular}{lll}
\hline MF & Heparin binding & 0.001728 \\
& Receptor binding & 0.003766 \\
& Calcium ion binding & 0.005090 \\
& Chemoattractant activity & 0.005946 \\
& Steroid hormone receptor activity & 0.007413 \\
& Chemotaxis & $6.15 \times 10^{-6}$ \\
BP & Cell-cell signaling & $9.42 \times 10^{-6}$ \\
& Positive regulation of endothelial cell proliferation & $3.98 \times 10^{-5}$ \\
& Positive regulation of mesenchymal cell proliferation & 0.000385 \\
& Skeletal system development & 0.000553 \\
CC & Extracellular region & $1.51 \times 10^{-8}$ \\
& Extracellular space & $5.45 \times 10^{-7}$ \\
& Proteinaceous extracellular matrix & $1.07 \times 10^{-6}$ \\
$1.96 \times 10^{-6}$ & Integral component of plasma membrane & 0.001295
\end{tabular}

DEGs, differentially expressed genes; PNI, perineural invasion; MF, molecular function, BP, biological process; CC, Cellular component.

Western blot analysis. Western blot analysis was performed as previously described (16) using the aforementioned cell lines and anti-FGF2 (1:1,000; cat. no. ab126861; Abcam). GAPDH (1:1,000; cat. no. KC-5G4; Kangchen BioTech Co., Ltd.) was used as an internal control. The protein level was quantified by the ImageJ (version 1.52k, National Institutes of Health) software. The mean levels for the high PNI ability group were compared with the mean level in the low ability group.

Data mining using the cancer genome atlas (TCGA). TCGA public database (hgserver1.amc.nl/cgi-bin/r2/main.cgi) was used to validate the association between FGF2 expression levels and overall survival. Specifically, the dataset 'Tumor Pancreatic adenocarcinoma, TCGA-178' was analyzed online. In total, 32 out of 178 patients were excluded due to incomplete follow-up data.

Statistical analysis. SPSS software (version 17.0; SPSS, Inc.) was used for statistical analysis. Graphical representations were constructed using GraphPad Prism (version 6; GraphPad
Software, Inc.). All data were presented as the mean \pm SD from three independent experiments. $\chi^{2}$ test was used to analyze the categorical variables. Numerical variables between two groups were assessed using two-tailed unpaired Student's t-test. In the analysis of variables between high and low PNI ability cells, the overall mean in each group was calculated, rather than for each individual cell line. The comparison of multiple groups was determined using parametric one-way ANOVA with Bonferroni's post hoc test. Survival curves were plotted using the Kaplan-Meier method, and differences were analyzed via the log-rank test using SPSS version 17.0 (IBM, Corp.). $\mathrm{P}<0.05$ (two-sided) was considered to indicate a statistically significant difference.

\section{Results}

PNI ability of cancer cell lines. Following dissection, nerve involvement was classified as running superficial of (Fig. 1A and C) or within (Fig. 1B and D) the tumor. The latter was considered to indicate PNI. PNI was observed in 
Table IV. A total of eight KEGG pathways are associated with PNI.

\begin{tabular}{|c|c|c|c|c|c|}
\hline Regulation & ID & KEGG pathway & Gene count & P-value & Genes \\
\hline Up & hsa04962 & $\begin{array}{l}\text { Vasopressin-regulated water } \\
\text { reabsorption }\end{array}$ & 3 & 0.044488 & DYNC1I1, CREB5, DYNC2H1 \\
\hline \multirow[t]{7}{*}{ Down } & hsa03320 & PPAR signaling pathway & 5 & 0.012359 & MMP-1, PCK1, FABP3, CD36, ACOX2 \\
\hline & hsa05200 & Pathways in cancer & 12 & 0.015915 & $\begin{array}{l}\text { CXCL12, GNGT1, FZD4, MMP-1, GLI1, AR, } \\
\text { FLT3, IGF1, CTNNA2, GNG4, FGFR1, FGF2 }\end{array}$ \\
\hline & hsa05144 & Malaria & 4 & 0.027847 & IL1B, HBB, CD36, KLRK1 \\
\hline & hsa05323 & Rheumatoid arthritis & 5 & 0.030419 & IL1B, CXCL12, MMP-1, TEK, CCL20 \\
\hline & hsa05032 & Morphine addiction & 5 & 0.033831 & PDE4D, GABRG2, PDE4B, GNGT1, GNG4 \\
\hline & hsa04060 & $\begin{array}{l}\text { Cytokine-cytokine receptor } \\
\text { interaction }\end{array}$ & 8 & 0.034815 & $\begin{array}{l}\text { IL1B, FLT3, CCL20, TNFSF9, CCR9, } \\
\text { TNFRSF19, CCR5, CXCL12 }\end{array}$ \\
\hline & hsa04062 & Chemokine signaling pathway & 7 & 0.038868 & $\begin{array}{l}\text { CCR5, CXCL12, CCL20, CCR9, CCL15, } \\
\text { GNGT1, GNG4 }\end{array}$ \\
\hline
\end{tabular}

KEGG, Kyoto Encyclopedia of Genes and Genomes; PNI, perineural invasion; DEGs, differentially expressed genes.

Table V. Hub genes associated with perineural invasion.

\begin{tabular}{llc}
\hline Gene & \multicolumn{1}{c}{ Description } & Degree \\
\hline AR & Androgen receptor & 32 \\
MAPT & Microtubule-associated protein $\tau$ & 15 \\
TEK & TEK receptor tyrosine kinase & 13 \\
SNCA & Synuclein $\alpha$ & 12 \\
UBE2E1 & Ubiquitin-conjugating enzyme E2 E1 & 12 \\
GLI1 & GLI-Kruppel family member GLI1 & 12 \\
FGFR1 & Fibroblast growth factor receptor 1 & 11 \\
ITPR1 & Inositol 1,4,5-trisphosphate receptor & 11 \\
& type 1 & \\
PRKG1 & Protein kinase, cGMP-dependent, type I & 11 \\
CCR5 & C-C motif chemokine receptor 5 & 10 \\
GNB4 & G protein subunit $\beta$ 4 & 10 \\
WWTR1 & WW domain-containing transcription & 10 \\
& regulator 1 & \\
PGR & Progesterone receptor & 8 \\
FGF2 & Fibroblast growth factor 2 & 7 \\
TNS1 & Tensin 1 & 7 \\
NPSR1 & Neuropeptide S receptor 1 & 7 \\
MITF & Melanogenesis-associated transcription & 6 \\
& factor & 6 \\
FLT3 & Fms-like tyrosine kinase 3 & 6 \\
\hline & Transforming growth factor $\beta$ 2 & 6 \\
\hline
\end{tabular}

all five mice injected with the PANC-1 cell line, whereas it was only observed in three mice injected with the CFPAC-1 and CAPAN-2 cell lines, and in two mice with the HPAC cells. By contrast, PNI was not observed in mice injected with the SW1990 or ASPC-1 cell lines. No tumor formation was observed in the HPDE control group. The cell lines were subsequently divided into two groups: High (comprising PANC-1, CFPAC and CAPAN-2) and low PNI ability group
Table VI. Top 10 KEGG pathways in protein-protein interaction network analysis.

\begin{tabular}{lrcc}
\hline & Number & & $\begin{array}{c}\text { False } \\
\text { discovery } \\
\text { rate }\end{array}$ \\
KEGG pathway & 189 & $5.50 \times 10^{-22}$ & $1.19 \times 10^{-19}$ \\
\hline $\begin{array}{l}\text { Chemokine signaling } \\
\text { pathway }\end{array}$ & 310 & $8.34 \times 10^{-21}$ & $9.05 \times 10^{-19}$ \\
Pathways in cancer & 87 & $6.16 \times 10^{-19}$ & $4.46 \times 10^{-17}$ \\
Prostate cancer & 70 & $4.60 \times 10^{-18}$ & $2.50 \times 10^{-16}$ \\
Adherens junction & 95 & $1.10 \times 10^{-15}$ & $4.78 \times 10^{-14}$ \\
Cholinergic synapse & 89 & $2.61 \times 10^{-14}$ & $8.08 \times 10^{-13}$ \\
Gap junction & 89 & $2.61 \times 10^{-14}$ & $8.08 \times 10^{-13}$ \\
Chagas disease (American & & & \\
trypanosomiasis) & 101 & $4.93 \times 10^{-13}$ & $1.34 \times 10^{-11}$ \\
Melanogenesis & 73 & $4.70 \times 10^{-12}$ & $1.13 \times 10^{-10}$ \\
Chronic myeloid leukemia & 94 & $8.21 \times 10^{-12}$ & $1.78 \times 10^{-10}$ \\
GnRH signaling pathway & 94 & \\
\hline KEGG, Kyoto Encyclopedia of Genes and Genomes. & \\
\hline
\end{tabular}

(comprising SW1990 and ASPC-1). Limb function of the mice in the high PNI group was significantly lower compared with that in the low PNI group (2.6 \pm 1.1 vs. $3.3 \pm 0.5 ; \mathrm{P}=0.036)$. Sciatic nerve function index and tumor length, width and volume were presented in Table I.

Identification of PNI-associated DEGs. Differential expression analysis of the two PNI ability groups identified 445 DEGs (fold-change $>1.50$ or $<0.66 ; \mathrm{P}<0.05$ ), among which 176 (39.6\%) were upregulated, and 269 (60.4\%) were downregulated in the high PNI group compared with the low PNI group. The top 10 genes in the up- and downregulated groups are presented in Table II. Sulfatase 2 (SULF2) has previously been reported to be associated with PNI (18), but the other genes 
Table VII. Validation results using 28 PNI and 22 non-PNI tumors in the GSE102238 dataset.

A, Top 10 DEGs (upregulated and downregulated)

\begin{tabular}{lcc}
\hline Gene & FC & P-value \\
\hline Upregulated DEGs & & \\
BNIP3 & 1.063 & 0.805 \\
EFEMP1 & 1.160 & 0.493 \\
GNB4 & 0.897 & 0.481 \\
NETO2 & 1.047 & 0.787 \\
LY75 & 0.880 & 0.414 \\
TSHZ3 & 1.212 & 0.282 \\
LIMS3 & N/A & N/A \\
TMSB15B & 1.118 & 0.486 \\
NAP1L5 & 1.116 & 0.400 \\
CLDN10 & 1.067 & 0.837 \\
Downregulated DEGs & & \\
SPON1 & 1.339 & 0.329 \\
CDH11 & 1.030 & 0.891 \\
SDPR & 1.169 & 0.485 \\
SOSTDC1 & 1.416 & 0.309 \\
LUM & 1.092 & 0.573 \\
IL1B & 0.851 & 0.524 \\
ESM1 & 1.141 & 0.667 \\
CCL20 & 0.730 & 0.406 \\
SULF2 & 0.996 & 0.978 \\
PDE4B & 0.763 & 0.188 \\
\hline & &
\end{tabular}

B, DEGs from KEGG pathways

\begin{tabular}{lcc}
\hline Gene & FC & P-value \\
\hline $\begin{array}{l}\text { Vasopressin-regulated water reabsorption } \\
\text { (upregulated) }\end{array}$ & & \\
DYNC1I1 & 1.081 & 0.584 \\
CREB5 & 1.059 & 0.708 \\
DYNC2H1 & 1.051 & 0.577 \\
PPAR signaling pathway (downregulated) & & \\
MMP1 & 0.583 & 0.172 \\
PCK1 & 0.922 & 0.830 \\
FABP3 & 0.870 & 0.368 \\
CD36 & 0.949 & 0.865 \\
ACOX2 & 1.053 & 0.509 \\
Pathways in cancer (downregulated) & & \\
CXCL12 & 1.061 & 0.794 \\
GNGT1 & 1.479 & 0.266 \\
FZD4 & 1.078 & 0.694 \\
GLI1 & 1.349 & 0.233 \\
FGFR1 & 1.196 & 0.243 \\
AR & 1.342 & 0.076 \\
FLT3 & 1.002 & 0.990 \\
IGF1 & 0.902 & 0.760 \\
CTNNA2 & 0.888 & $0.049^{\mathrm{a}}$ \\
GNG4 & 1.094 & 0.619 \\
FGF2 & 1.451 & $0.038^{\mathrm{a}}$ \\
& &
\end{tabular}

Table VII. Continued.

\begin{tabular}{lcc}
\hline C, Hub genes & & \\
\hline Genes & FC & P-value \\
\hline FGF2 & 1.451 & $0.038^{a}$ \\
GLI1 & 1.349 & 0.233 \\
NPSR1 & 0.942 & 0.914 \\
CCR5 & 1.012 & 0.957 \\
PDE4D & 0.919 & 0.664 \\
TEK & 0.966 & 0.817 \\
FGFR1 & 1.196 & 0.243 \\
PGR & 1.237 & 0.239 \\
TNS1 & 1.275 & 0.074 \\
MAPT & 1.316 & 0.082 \\
AR & 1.342 & 0.076 \\
FLT3 & 1.002 & 0.990 \\
GNB4 & 0.897 & 0.481 \\
UBE2E1 & 0.997 & 0.974 \\
WWTR1 & 1.210 & 0.114 \\
TGFB2 & 1.262 & 0.304 \\
PRKG1 & 1.138 & 0.377 \\
SNCA & 1.012 & 0.944 \\
ITPR1 & 0.986 & 0.911 \\
MITF & 0.946 & 0.598 \\
\hline
\end{tabular}

${ }^{\mathrm{a}} \mathrm{P}<0.05$. PNI, perineural invasion; DEGs, differentially expressed genes; FC, fold-change; Kyoto Encyclopedia of Genes and Genomes.

were not be reported previously. And the top 10 upregulated and downregulated genes were selected for further validation.

Functional annotation of PNI-associated DEGs. The significantly enriched GO terms were ranked according to the $\mathrm{P}$-value. The top five ranked terms for the three different GO sub-ontologies (cellular component, biological process, and molecular function) are presented in Table III. The results of KEGG pathway enrichment analysis for the up- and downregulated genes are presented in Table IV. A total of eight pathways exhibited significant differences $(\mathrm{P}<0.05)$. Upregulated genes were involved in one pathway, whereas the downregulated genes were involved in seven pathways. Considering the total number of genes, 19 differential genes from two downregulated and one upregulated pathways were selected for further validation. Matrix metalloproteinase-1 (MMP-1) (19) and C-X-C motif chemokine ligand 12 (CXCL12) (20,21) have been previously reported to be associated with PNI.

Candidate hub genes associated with PNI. The 445 PNI-associated DEGs were regarded as seed proteins and introduced into the NetworkAnalyst database for PPI network analysis. Due to the large amount of seed proteins, the mode of zero-order interactors was selected, as aforementioned. A total of four sub-networks, 265 nodes, 434 edges and 131 seeds were identified. A total of 20 genes with gene degree $>6$ were regarded as hub genes (Table V) and were selected for further 
A

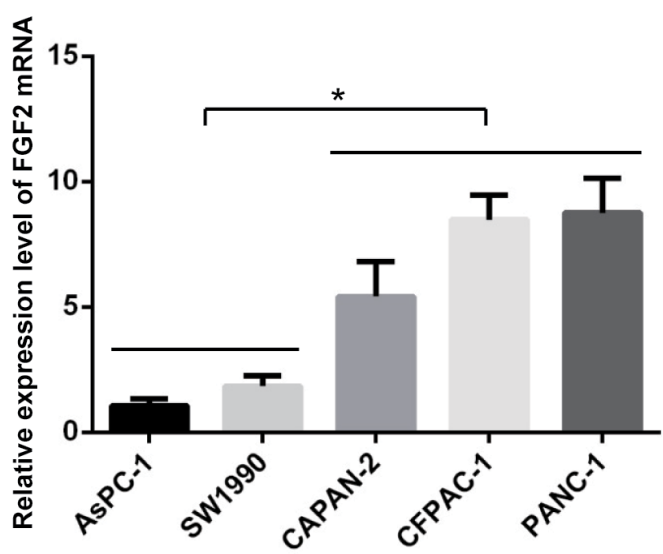

B

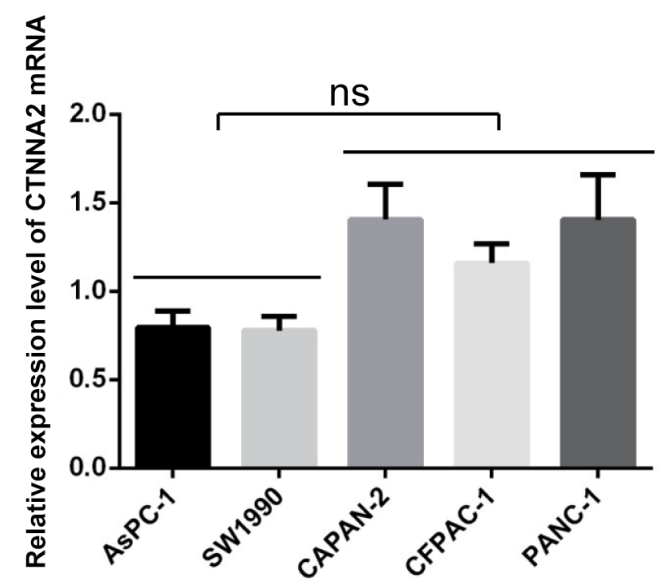

C
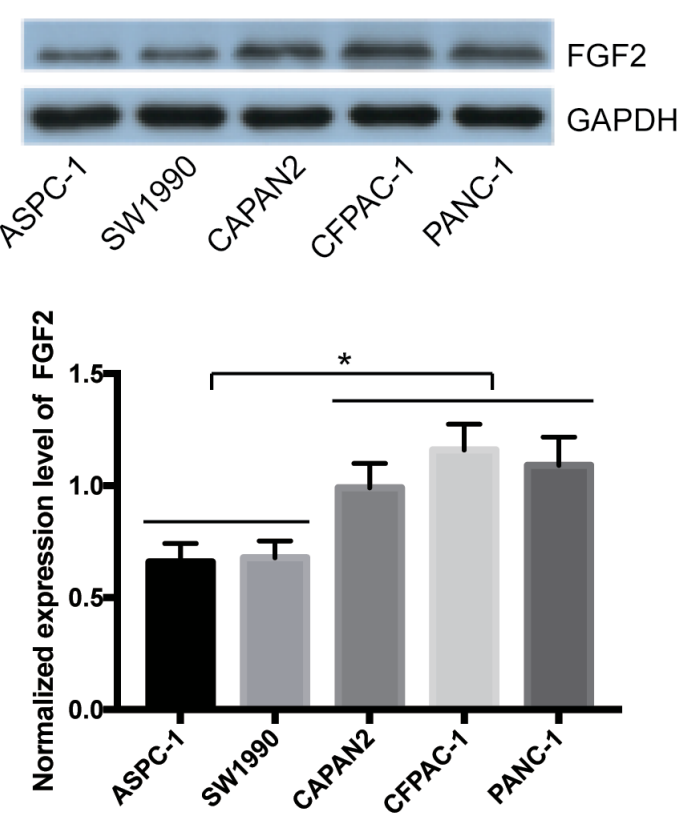

Figure 2. Expression levels of FGF2 and CTNNA2 in pancreatic cancer cells. The expression levels of (A) FGF2 and (B) CTNNA2 were determined the mRNA level using reverse transcription-quantitative PCR. (C) Expression levels of FGF2 at the protein level were determined using western blot analysis. Black lines indicate different groups. Data are presented as the mean $\pm \mathrm{SD}(\mathrm{n}=3)$. ${ }^{*} \mathrm{P}<0.05$. FGF2, fibroblast growth factor 2; CTNNA2, catenin $\alpha 2$; ns, not significant.

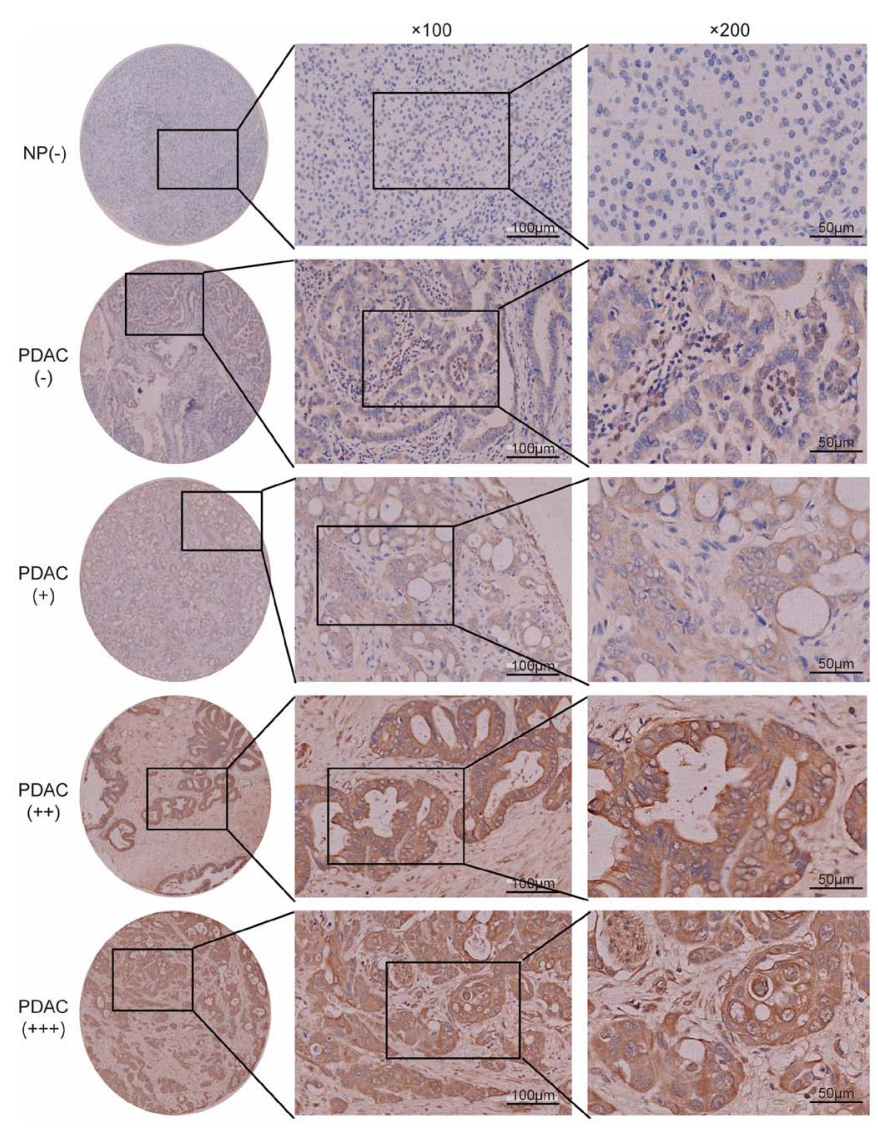

Figure 3. Expression level of FGF2 in PDAC tissue. Representative images of immunohistochemical staining of FGF2 in NP and PDAC tissue (magnification, x100 and x200, respectively. Representative images of FGF2 were classified as negative (-), weak (+), moderate $(++)$ or strong staining (+++). NP, normal pancreas; FGF2, fibroblast growth factor 2; PDAC, pancreatic ductal adenocarcinoma.

validation. C-C chemokine receptor type 5 (CCR5) has previously been associated with PNI (22). For genes identified in the PPI subnetwork, the top two pathways were 'Chemokine signaling pathway' and 'Pathways in cancer' (Table VI), which were also identified in the downregulated KEGG pathway (Table IV).

Validation of DEGs and hub genes in GSE102238. As described above, we adopted three methods to select the genes associated with PNI. The first group included the top 10 upregulated and downregulated genes from differential expression analysis of the two PNI ability groups. The second group included 19 differential genes from two downregulated (hsa03320: PPAR signaling pathway; hsa05200: Pathways in cancer) and one upregulated (hsa04962: Vasopressin-regulated water reabsorption) KEGG pathways. The third group included 20 hub genes from PPI network analysis. The genes were validated by comparing 28 PNI and 22 non-PNI tumors in the GSE102238 dataset (Table VII). These results demonstrated that only two genes, FGF2 and CTNNA2, were differently expressed in GSE102238.

Validation of protein and mRNA expression levels. The expression levels of FGF2 and CTNNA2 in the pancreatic cancer cell lines were subsequently validated. The mean levels for the high PNI ability group were compared with the mean levels in the low 
Table VIII. Association between FGF2 expression levels and clinicopathological characteristics in patients with pancreatic ductal adenocarcinoma.

Expression level of FGF2

\begin{tabular}{|c|c|c|c|c|}
\hline \multirow{2}{*}{ Clinicopathological characteristic } & \multirow{2}{*}{ Total $(n=157)$} & & & \multirow{2}{*}{ P-value } \\
\hline & & Positive, $\mathrm{n}=31(19.7 \%)$ & Negative, $n=126(80.3 \%)$ & \\
\hline Age, years, n (\%) & & & & 0.332 \\
\hline$\leq 65$ & 73 & $12(16.4)$ & $61(83.6)$ & \\
\hline$>65$ & 84 & $19(22.6)$ & $65(77.4)$ & \\
\hline Sex, n (\%) & & & & 0.191 \\
\hline Male & 90 & $21(23.3)$ & 69 (76.7) & \\
\hline Female & 67 & $10(14.9)$ & $57(85.1)$ & \\
\hline Tumor size, cm, n (\%) & & & & 0.201 \\
\hline$\leq 2.0$ & 30 & $3(10.0)$ & $27(90.0)$ & \\
\hline$>2.0$ & 127 & $28(22.0)$ & $99(78.0)$ & \\
\hline Tumor location, $\mathrm{n}(\%)$ & & & & 0.628 \\
\hline Head & 107 & $20(18.7)$ & $87(81.3)$ & \\
\hline Body/tail & 50 & $11(22.0)$ & $39(78.0)$ & \\
\hline Tumor differentiation, $\mathrm{n}(\%)$ & & & & $0.013^{\mathrm{b}}$ \\
\hline Well/moderate & 101 & $14(13.9)$ & $87(86.1)$ & \\
\hline Poor & 56 & $17(30.4)$ & 39 (69.6) & \\
\hline Perineural invasion, $\mathrm{n}(\%)$ & & & & $0.013^{\mathrm{b}}$ \\
\hline Absent & 44 & $3(6.8)$ & $41(93.2)$ & \\
\hline Present & 113 & $28(24.8)$ & $85(75.2)$ & \\
\hline Nodal metastasis, n (\%) & & & & 0.976 \\
\hline Absent & 106 & $21(19.8)$ & $85(80.2)$ & \\
\hline Present & 51 & $10(19.6)$ & $41(80.4)$ & \\
\hline Lymphovascular invasion, $\mathrm{n}(\%)$ & & & & 0.843 \\
\hline Absent & 135 & $27(20.0)$ & $108(80.0)$ & \\
\hline Present & 22 & $4(18.2)$ & $18(81.8)$ & \\
\hline Clinical stage, $\mathrm{n}(\%)$ & & & & $0.046^{\mathrm{b}}$ \\
\hline $\mathrm{I} / \mathrm{II}$ & 118 & $19(16.1)$ & $99(83.9)$ & \\
\hline III & 39 & $11(28.2)$ & $28(71.8)$ & \\
\hline
\end{tabular}

${ }^{\mathrm{a}} \chi^{2}$ test. ${ }^{\mathrm{b}} \mathrm{P}<0.05$. FGF2, fibroblast growth factor 2 .

ability group. FGF2 mRNA expression levels were significantly higher in the high PNI ability group compared with that in the low PNI ability group (Fig. 2A). CTNNA2 mRNA expression levels were not significantly different between the two groups (Fig. 2B). The protein expression level of FGF2 was also higher in the high PNI ability group, using western blot analysis (Fig. 2C). FGF2 was selected for validation using tissue microarray analysis. Epithelial cells in normal pancreas exhibited low FGF2 expression levels (Fig. 3). None of the non-tumor tissues showed high FGF2 expression levels. In patients with PDAC, $31(19.7 \%)$ cases exhibited a high expression level of FGF2, and $126(80.3 \%)$ cases exhibited a low FGF2 expression level (Fig. 3). FGF2 expression levels in PDAC were associated with PNI, tumor differentiation and TNM stage (Table VIII). Patients with high expression levels of FGF2 had a significantly worse survival rate compared with those with low expression levels [12.2 months; 95\% confidence interval (CI), 7.1-17.3 months, vs. 21.9 months; 95\% CI, 18.5-25.4 months; $\mathrm{P}<0.001$ ] (Fig. 4A).
TCGA public database (Tumor Pancreatic adenocarcinoma, TCGA-178) was used to validate the association between FGF2 expression levels and overall survival online. In TCGA public database, 146 patients were followed up. And the patients were divided into the high and low expression groups in a similar proportion with Renji cohort. The results demonstrated that a high expression level of FGF2 was associated with poor survival (Fig. 4B).

\section{Discussion}

Identifying the molecular characteristics of PNI is key for a greater understanding of tumor biology. Previous studies have used pancreatic cancer cells $(6,7)$ to distinguish PNI-associated genes in cell lines with high or low PNI ability. In the present study, pancreatic cancer cells were also used to identify PNI-associated genes. To the best of our knowledge, the present study is the first to use functional enrichment and 
A

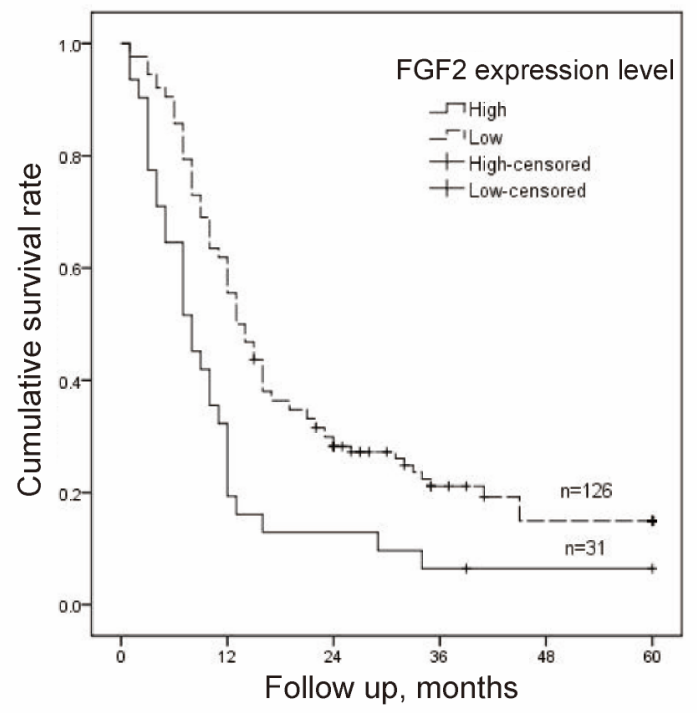

B

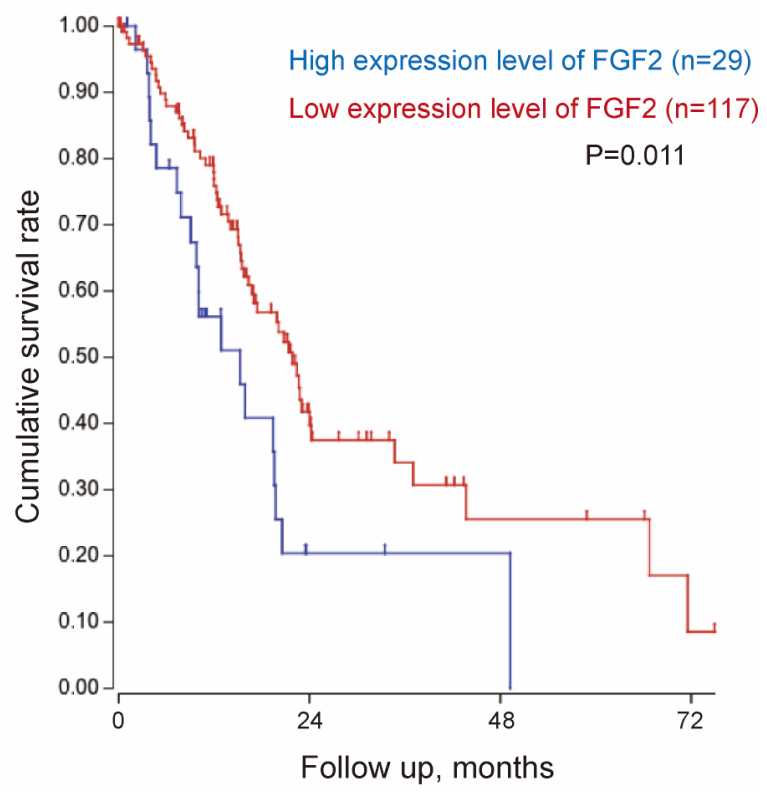

Figure 4. Expression levels of FGF2 and OS (A) Kaplan-Meier survival curves demonstrating that $\mathrm{OS}$ of patients with high expression levels of FGF2 was significantly shorter vs. patients with low expression levels of FGF2. (B) Kaplan-Meier survival curves demonstrating that OS of patients with high expression levels of FGF2 was significantly shorter compared with patients with low expression levels of FGF2 in The Cancer Genome Atlas cohort. 146 patients were followed up. A total of 32 out of 178 patients were excluded due to incomplete follow-up data. OS, overall survival; FGF2, fibroblast growth factor 2 .

PPI network analysis to distinguish between PNI-associated pathways and hub genes.

In the present study, 'chemokine signaling pathway' was the top pathway associated with PNI in the PPI network. A total of four genes (SULF2, MMP-1, CXCL12 and CCR5) from identified DEGs and hub genes have been reported to be associated with PNI (18-22). Of these genes, CXCL12 and CCR5 were found to be involved in the 'chemokine signaling pathway'. The CXCL12/CXCR4 axis is a widely studied chemokine signaling pathway in cancer (23). It has been reported (20) that CXCL12 is associated with PNI in pancreatic cancer; CXCL12 is secreted by peripheral nerves and stimulates chemotactic migration of CXCR4(+) cancer cells in a paracrine manner, which results in PNI. Similarly, CXCR4(+) breast cancer cells migrate toward distant organs, such as lung and liver, in response to a CXCL12 gradient (24). CCR5 is the chemokine receptor for C-C motif chemokine ligand 5 (CCL5), which is an inflammatory chemokine (25). A previous study (22) demonstrated that exogenous CCL5 significantly facilitated PNI activity in human salivary adenoid cystic carcinoma, which could be blocked by a CCR5 inhibitor (e.g., maraviroc). In addition, CCL5 is secreted by Schwann and dorsal root ganglia cells (26). These studies demonstrate that the 'chemokine signaling pathway' is associated with PNI. Chemokine receptors, which are aberrantly expressed (increased or decreased) on the cancer cell surface, are potential therapeutic targets as well as a possible method for identification of PNI-associated genes.

FGF2, also known as basic FGF, is part of the 22-member FGF family (27). It is a multifunctional growth factor that exerts potent angiogenic effects and serves a key role in the differentiation and function of the central nervous system $(26)$. A number of studies $(28,29)$ have demonstrated that high expression levels of FGF2 are significant prognostic indicators for patients with PDAC. However, to the best of our knowledge, there have been no previous studies regarding the association between FGF2 and PNI. In the present study, FGF2 was demonstrated to be a hub gene associated with PNI in pancreatic cancer cell lines, and further validated by a tissue microarray. It has been reported that high levels of FGF2 are detected from neurulation onwards during development. Moreover, developmental expression of FGF2 and its receptors is temporally and spatially regulated (27). Therefore, an increased FGF2/FGFR1 signaling is a focus of therapeutic development for neurodegenerative disorders, due to its importance in adult neurogenesis and neuroinflammation (27), and FGF2 may be a potential target in the treatment of pancreatic cancer.

In conclusion, the present study identified PNI-associated genes in pancreatic cancer cell lines and the 'chemokine signaling pathway' was found to be associated with PNI, following KEGG pathway enrichment analysis and the construction of a PPI network from the identified DEGs. Furthermore, FGF2 was found to be associated with PNI. The present study provides a potential method to identify PNI-associated genes. However, further investigation of PNI-associated genes is required to elucidate the molecular mechanisms underlying PNI, and to identify potential therapeutic targets.

\section{Acknowledgements}

Not applicable.

\section{Funding}

The present study was supported by grants from the National Natural Science Foundation of China (grant nos. 81401931, 81702739, 81602414 and 81802317), Science and Technology Funds of the School of Medicine, Shanghai Jiao Tong 
University (grant no. 14XJ10022), the Scientific Research Project of Shanghai Municipal Health and Family Planning Commission (grant no. 201740218) and Shanghai Minhang Science and Technology Committee (grant no. 2018MHZ063).

\section{Availability of data and materials}

The datasets used and/or analyzed during the current study are available from the corresponding author on reasonable request.

\section{Authors' contributions}

JZ, YS, JY and JW conceived and designed the study, collected and analyzed the data. JZ and DL wrote the original manuscript and revised it for important intellectual content. XF and DL performed the experiments and collected and analyzed the data. RH also analyzed the data. MY performed the experiments and revised the manuscript. JY, JW and RH revised the manuscript. YH and WL collected and analyzed the data. All authors read and approved the final manuscript.

\section{Ethics approval and consent to participate}

The animal studies were performed at East China Normal University. All animal experiments were performed in accordance with the NIH Guide for the Care and Use of Laboratory Animal studies was approved by the Research Ethics Committee of East China Normal University (approval no. 20141204). The Human PDAC tissue microarray containing 205 cases of tumor and matched non-tumor tissues from Renji Hospital, School of Medicine, Shanghai Jiao Tong University. The follow-up data included postoperative treatment, tumor marker and imaging examination, such as CT, MRI and PET-CT. The human experiments of the present study were approved by the Research Ethics Committee of Renji Hospital, School of Medicine, Shanghai Jiao Tong University (Shanghai, China).

\section{Patient consent for publication}

Written informed consent was provided for publication by all patients prior to enrolment.

\section{Competing interests}

The authors declare that they have no competing interests.

\section{References}

1. Liebig C, Ayala G, Wilks JA, Berger DH and Albo D: Perineura invasion in cancer: A review of the literature. Cancer 115: 3379-3391, 2009.

2. Bapat AA, Hostetter G, Von Hoff DD and Han H: Perineural invasion and associated pain in pancreatic cancer. Nat Rev Cancer 11: 695-707, 2011.

3. Zhang JF, Hua R, Sun YW, Liu W, Huo YM, Liu DJ and Li J: Influence of perineural invasion on survival and recurrence in patients with resected pancreatic cancer. Asian Pac J Cancer Prev 14: 5133-5139, 2013.

4. Zhu Z, Friess H, diMola FF, Zimmermann A, Graber HU, Korc M and Büchler MW: Nerve growth factor expression correlates with perineural invasion and pain in human pancreatic cancer. J Clin Oncol 17: 2419-2428, 1999.
5. Stopczynski RE, Normolle DP, Hartman DJ, Ying H, DeBerry JJ Bielefeldt K, Rhim AD, DePinho RA, Albers KM and Davis BM: Neuroplastic changes occur early in the development of pancreatic ductal adenocarcinoma. Cancer Res 74: 1718-1727, 2014.

6. Koide N, Yamada T, Shibata R, Mori T, Fukuma M, Yamazaki K, Aiura K, Shimazu M, Hirohashi S, Nimura Y and Sakamoto M: Establishment of perineural invasion models and analysis of gene expression revealed an invariant chain (CD74) as a possible molecule involved in perineural invasion in pancreatic cancer. Clin Cancer Res 12: 2419-2426, 2006.

7. Abiatari I, DeOliveira T, Kerkadze V, Schwager C, Esposito I, Giese NA, Huber P, Bergman F, Abdollahi A, Friess H and Kleeff J: Consensus transcriptome signature of perineural invasion in pancreatic carcinoma. Mol Cancer Ther 8: 1494-1504, 2009.

8. Gil Z, Cavel O, Kelly K, Brader P, Rein A, Gao SP, Carlson DL, Shah JP, Fong Y and Wong RJ: Paracrine regulation of pancreatic cancer cell invasion by peripheral nerves. J Natl Cancer Inst 102: 107-118, 2010.

9. Shain AH, Giacomini CP, Matsukuma K, Karikari CA, Bashyam MD, Hidalgo M, Maitra A and Pollack JR: Convergent structural alterations define SWItch/Sucrose NonFermentable (SWI/SNF) chromatin remodeler as a central tumor suppressive complex in pancreatic cancer. Proc Natl Acad Sci USA 109: E252-E259, 2012.

10. R Core Team. R: A language and environment for statistical computing. $\mathrm{R}$ foundation for statistical computing. Vienna, Austria, ISBN 3-900051-07-0, 2012. URL http://www.R-project. org/.

11. Yang MW, Tao LY, Jiang YS, Yang JY, Huo YM, Liu DJ, Li J, $\mathrm{Fu} \mathrm{XL}, \mathrm{He} \mathrm{R}$, Lin $\mathrm{C}$, et al: Perineural invasion reprograms the immune microenvironment through cholinergic signaling in pancreatic ductal adenocarcinoma. Cancer Res 80: 1991-2003, 2020.

12. Xia J, Gill EE and Hancock RE: NetworkAnalyst for statistical, visual and network-based meta-analysis of gene expression data. Nat Protoc 10: 823-844, 2015.

13. Xia J, Benner MJ and Hancock RE: NetworkAnalyst-integrative approaches for protein-protein interaction network analysis and visual exploration. Nucleic Acids Res 42 (Web Server Issue): W167-W174, 2014

14. Yang JY, Jiang SH, Liu DJ, Yang XM, Huo YM, Li J, Hua R, Zhang ZG and Sun YW: Decreased LKB1 predicts poor prognosis in pancreatic ductal adenocarcinoma. Sci Rep 5: 10575, 2015.

15. Amin MB, Edge S, Greene F (eds), et al: AJCC cancer staging manual. 8th edition, New York, Springer, 2017.

16. Yan TT, Fu XL, Li J, Bian YN, Liu DJ, Hua R, Ren LL, Li CT, Sun YW, Chen HY, et al: Downregulation of RPL15 may predict poor survival and associate with tumor progression in pancreatic ductal adenocarcinoma. Oncotarget 6: 37028-37042, 2015.

17. Livak KJ and Schmittgen TD: Analysis of relative gene expression data using real-time quantitative PCR and the 2(-Delta Delta C(T)) method. Methods 25: 402-408, 2001.

18. Alhasan SF, Haugk B, Ogle LF, Beale GS, Long A, Burt AD, Tiniakos D, Televantou D, Coxon F, Newell DR, et al: Sulfatase-2: A prognostic biomarker and candidate therapeutic target in patients with pancreatic ductal adenocarcinoma. Br J Cancer 115: 797-804, 2016.

19. Kemik O, Kemik AS, Sümer A, Dulger AC, Adas M, Begenik H, Hasirci I, Yilmaz O, Purisa S, Kisli E, et al: Levels of matrix metalloproteinase-1 and tissue inhibitors of metalloproteinase-1 in gastric cancer. World J Gastroenterol 17: 2109-2112, 2011.

20. Xu Q, Wang Z, Chen X, Duan W, Lei J, Zong L, Li X, Sheng L, Ma J, Han L, et al: Stromal-derived factor-1 $\alpha /$ CXCL12-CXCR4 chemotactic pathway promotes perineural invasion in pancreatic cancer. Oncotarget 6: 4717-4732, 2015.

21. Zhang S, Qi L, Li M, Zhang D, Xu S, Wang N and Sun B: Chemokine CXCL12 and its receptor CXCR4 expression are associated with perineural invasion of prostate cancer. J Exp Clin Cancer Res 27: 62, 2008.

22. Shen Z, Li T, Chen D, Jia S, Yang X, Liang L, Chai J, Cheng X, Yang $X$ and Sun M: The CCL5/CCR5 axis contributes to the perineural invasion of human salivary adenoid cystic carcinoma. Oncol Rep 31: 800-806, 2014.

23. Teicher BA and Fricker SP: CXCL12 (SDF-1)/CXCR4 pathway in cancer. Clin Cancer Res 16: 2927-2931, 2010.

24. Müller A, Homey B, Soto H, Ge N, Catron D, Buchanan ME, McClanahan T, Murphy E, Yuan W, Wagner SN, et al: Involvement of chemokine receptors in breast cancer metastasis. Nature 410: 50-56, 2001. 
25. Aldinucci D and Colombatti A: The inflammatory chemokine CCL5 and cancer progression. Mediators Inflamm 2014: 292376, 2014.

26. Bolin LM, Murray R, Lukacs NW, Strieter RM, Kunkel SL, Schall TJ and Bacon KB: Primary sensory neurons migrate in response to the chemokine RANTES. J Neuroimmunol 81: 49-57, 1998.

27. Woodbury ME and Ikezu T: Fibroblast growth factor-2 signaling in neurogenesis and neurodegeneration. J Neuroimmune Pharmacol 9: 92-101, 2014

28. Kuwahara K, Sasaki T, Kuwada Y, Murakami M, Yamasaki S and Chayama K: Expressions of angiogenic factors in pancreatic ductal carcinoma: A correlative study with clinicopathologic parameters and patient survival. Pancreas 26: 344-349, 2003.
29. Ohta T, Yamamoto M, Numata M, Iseki S, Tsukioka Y, Miyashita T, Kayahara M, Nagakawa T, Miyazaki I, Nishikawa K and Yoshitake Y: Expression of basic fibroblast growth factor and its receptor in human pancreatic carcinomas. Br J Cancer 72: 824-831, 1995.

cc) (i) $\Theta$ This work is licensed under a Creative Commons Attribution-NonCommercial-NoDerivatives 4.0 International (CC BY-NC-ND 4.0) License. 